\title{
PRIMERAS OBSERVACIONES SOBRE EL I.U.D. EN COLOMBIA
}

\author{
INFORME PRELIMINAR SOBRE EL ANALISIS DE 1.000 HISTORIAS CLINICAS
}

DEL CENTRO DE PLANIFICACION FAMILIAR, UNIDAD PILOTO, DE BOGOTA, D. E.

\section{Dr. Germán Riaño Gamỏoa*}

A continuación se presenta un informe estadístico sobre el análisis de 1.000 historias clínicas ( 1.084 a 2.083) del Centro de Planificación Familiar, Unidad Piloto, correspondiente a los períodos Agosto-Diciembre de 1966, por lo tanto los datos obtenidos solamente son válidos por un espacio de 4 meses hasta el mes de Diciembre de 1966, fecha en que se efectuó el corte estadístico.

\section{Estructura por Edades}

Las edades de las pacientes estudiadas oscilaron entre los 15 y 50 años, con una edad promedio de 30.01 ; los mayores grupos correspondieron a las edades de 30 a 34 años; 25 a 29 y 20 a 24, según tabla adjunta (Ver Tabla № 1). Los grupos de menor incidencia correspondieron a las edades de 15 a 19 años y de más de 45.

\section{Clasificación Económica}

Los criterios para clasificación económica se basaron en el ingreso familiar (Marido y Mujer), durante el mes y lo hemos dividido en tres clases: $A, B, C$.

El grupo " $A$ " corresponde a aquel grupo de mujeres cuyo ingreso fa- miliar total mensual está por debajo de los \$800,00; estas pacientes pagan una cuota total de ingreso que oscila entre $\$ 10,00$ y $\$ 40,00$; cubre los gastos correspondientes a Examen de Citología Vaginal Tumoral y/o Biopsias, aplicación del dispositivo y cuatro controles por año. En caso de ser otro el método escogido se prestan iguales servicios. En el análisis correspondieron a este grupo 883 mujeres, o sea, el $88,3 \%$.

El grupo " $B$ " corresponde a aquel grupo de mujeres cuyo ingreso familiar mensual oscila entre los $\$ 800$,00 y $\$ 1.600,00$; la cuota de ingreso fijada para este grupo social varía entre $\$ 40,00$ y $\$ 80,00$. Cubre, como en el caso anterior, los casos correspondientes a lo anotado. En este grupo se encontraron 101 mujeres, o sea, el 10,1\%.

El grupo " $\mathrm{C}$ " incluye aquel grupo de mujeres cuyo ingreso familiar mensual está por encima de los $\$ 1$.600,00 ; en este grupo la cuota de ingreso oscila entre los $\$ 80,00$ y $\$$ 300,00 ; en el análisis se encontraron 14 mujeres correspondiendo a $0,1 \%$.

* Coordinador general del Centro de Planificación Familiar. 

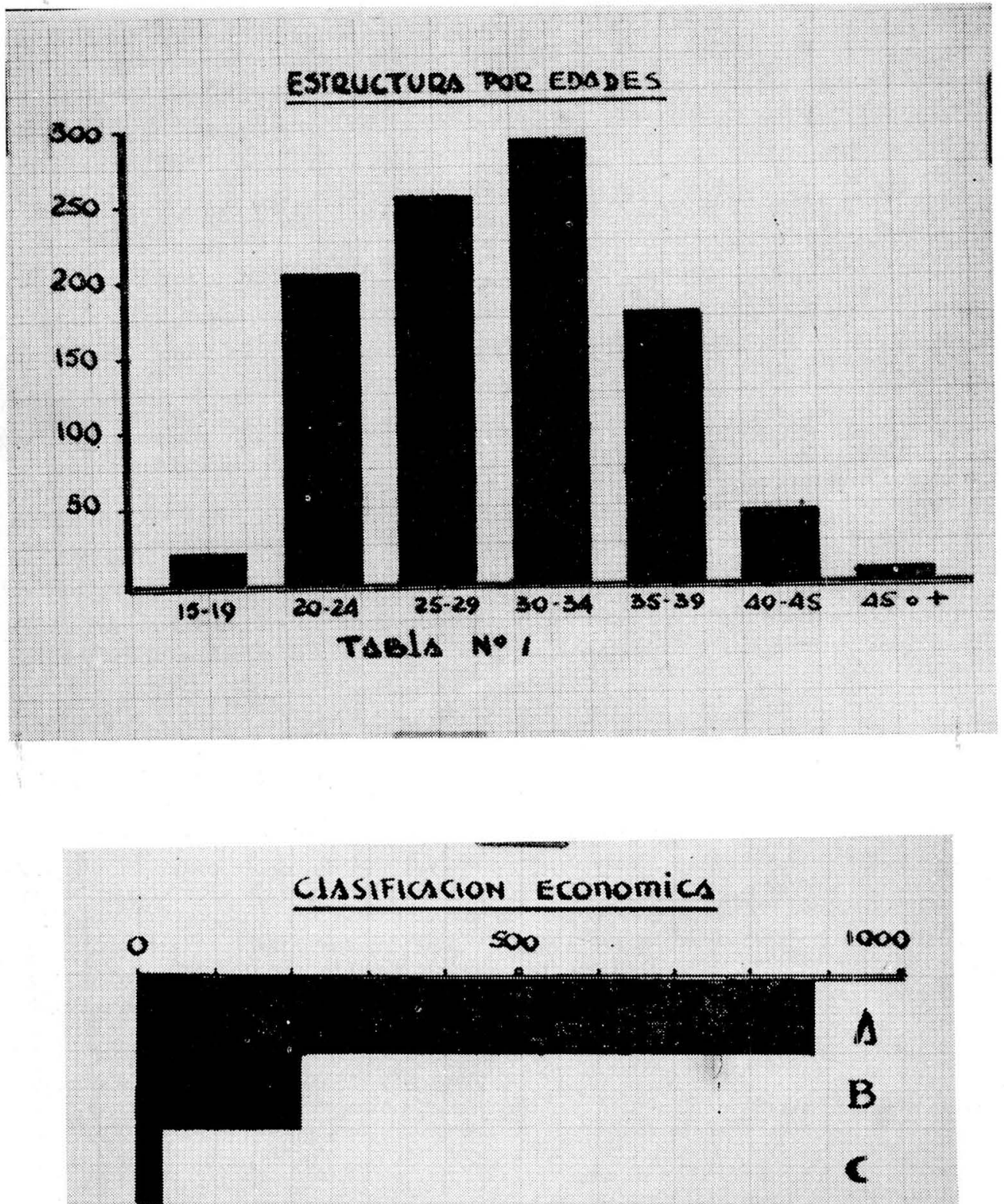

TABIS N.2 


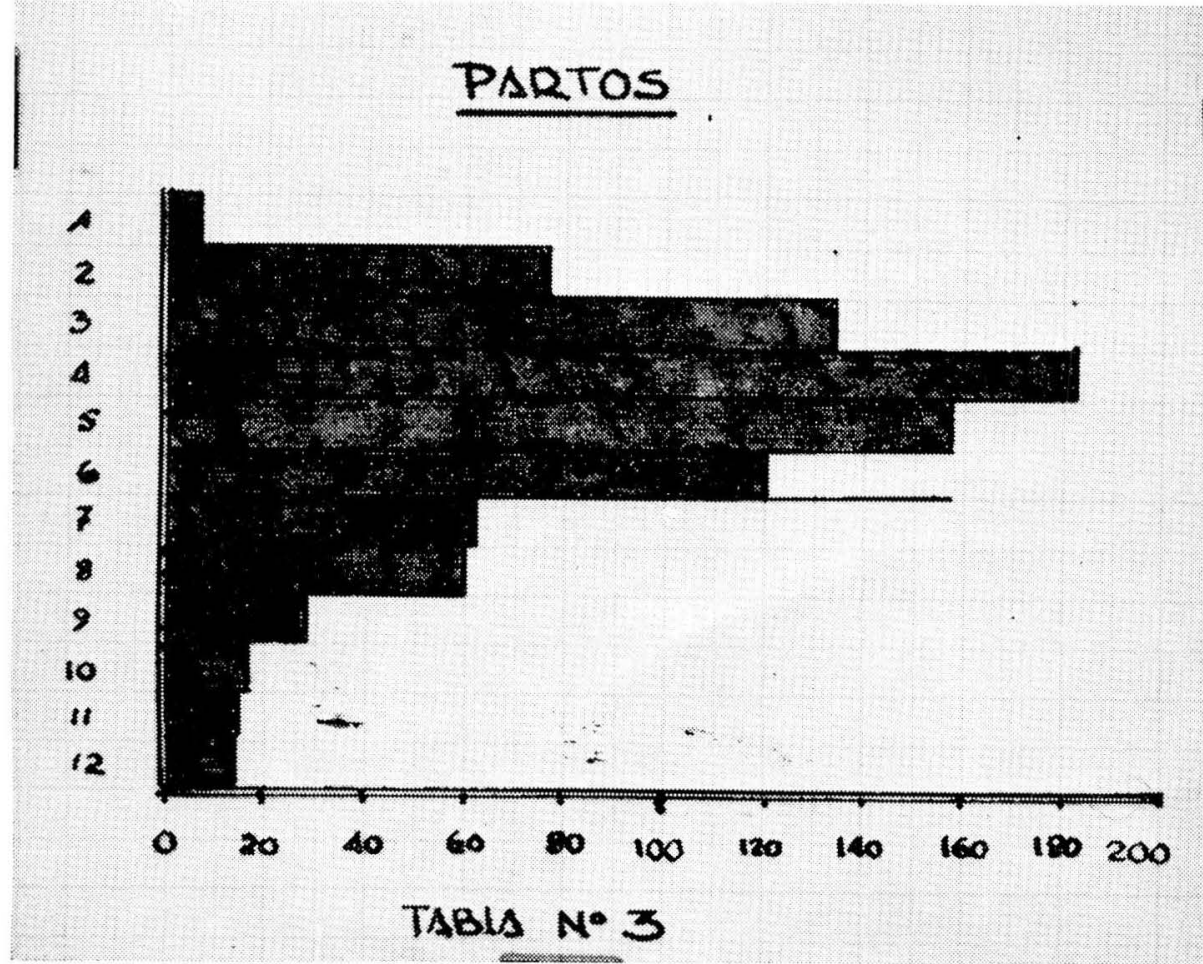

\section{Paridad}

La suma del número total de partos correspondiente a las $1.000 \mathrm{mu}-$ jeres del estudio, dió un total de 5.402 con un promedio de 5,4 partos por mujer. En otros estudios semejantes con diferentes grupos de mujeres el promedio de partos solo varió del 0.1 al 0.3 .

En cuanto a la distribución del número de partos en este grupo, se vió que el mayor grupo de mujeres había tenido 4 partos y el menor grupo 1 parto. El mayor número de partos encontrados fué de 16. (Ver Tabla No 3).

\section{Abortos}

Al revisar el número de abortos se encontró un total de 743 , lo cual dió un promedio de 0.7 abortos por mu- jer. La distribución del número de abortos por mujer se observa en la siguiente tabla. (Ver Tabla № 4).

Confesaron espontáneamente aborto provocado 63 mujeres de las 1.000, maniobra en casi todos los casos realizada con sonda; es de anotar que este mismo número de pacientes en controles sucesivos dió mayores detalles que serán objeto de un trabajo posterior (contribución al estudio de aborto provocado en Bogotá).

\section{Edad del último hijo}

Para este estudio se formaron tres grupos así: hasta de un año de nacido; de uno a dos años de nacido y de dos a tres años de nacido. En 421 pacientes la edad del último hijo fué de menos de un año;; de uno a dos años, 314 pacientes y de dos 


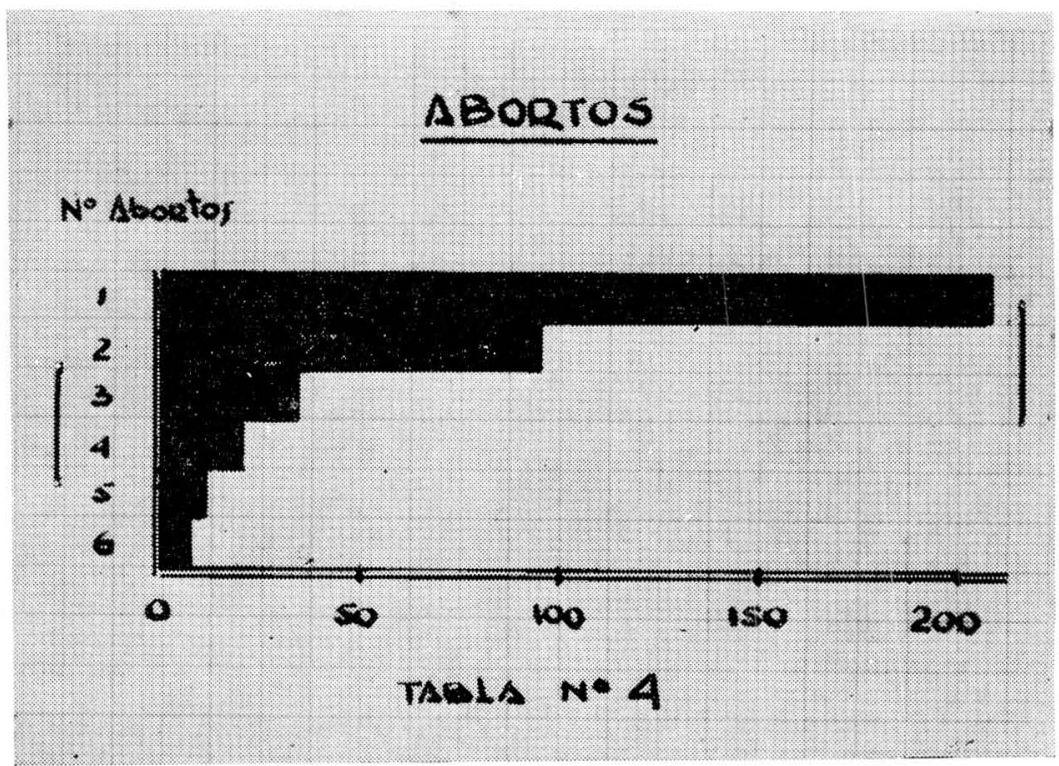

a tres años 264 pacientes. (Ver Tabla № 5 ).

\section{Anticonceptivos usados:}

El análisis sobre qué tipo de método habían usado o usaba en la actualidad este grupo de mujeres, dió los siguientes resultados:

\begin{tabular}{lrr}
\hline & & $\%$ \\
Tabletas orales: & 393 & 39.3 \\
Ovulos vaginales: & 26 & 2.6 \\
Coito interrupto: & 46 & 4.6 \\
Duchas vaginales: & 28 & 2.8 \\
Preservativo: & 28 & 2.8 \\
Diafragma: & 4 & .4 \\
Ritmo: & 3 & .3 \\
Desconocen método alguno: & 482 & 48.2 \\
Otros: & 19 & 1.9 \\
\hline
\end{tabular}

Analizando detenidamente estos datos obtenidos en forma retrospectiva se ve que, a pesar de la impresión casi general de que los grupos menos favorecidos desconocen método alguno tendiente al control de su fertilidad $y$, si es cierto el dato so- cio-económico obtenido de que se trabaja con el grupo "A" preferencialmente, $88,5 \%$, vemos que por lo menos la mitad de estas pacientes usan o usaron anovulatorios orales con fines anticonceptivos. El método que se piensa sea el más usado, coito interrupto o retiro) o era desconocido para el mayor grupo de mujeres o simplemente no se usaba con conocimiento de causa por parte de la mujer, como se vió en posteriores encuestas.

Los métodos menos favorecidos fueron los más costosos (diafragma) - los que requerían educación, voluntad y entrenamiento especial (continencia periódica o ritmo). La mitad del grupo en estudio no conocía ni había oído hablar de método alguno (482). También se encontró un pequeño grupo de mujeres que practicaba la anticoncepción con diferentes métodos pero en forma indiscriminada y alternándolos entre sí. 


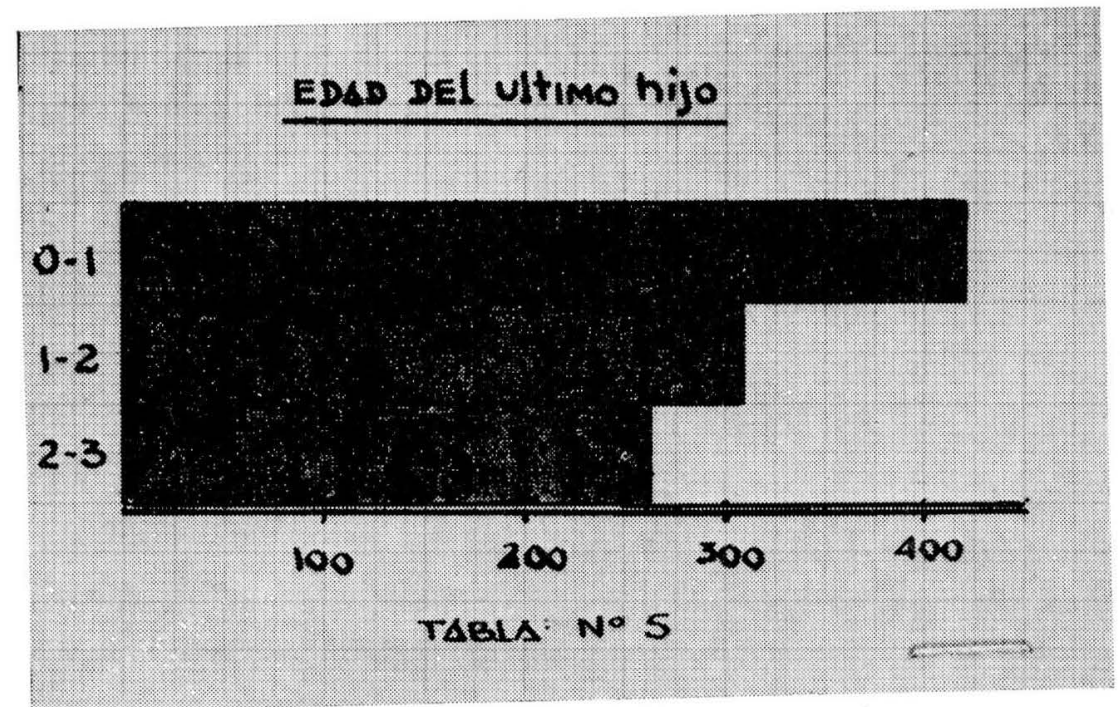

En el grupo denominado "Otros" se incluyen métodos "folclóricos", como fueron: mejoral en pastillas disueltas o intravaginales, jugo de limón o trozos intravaginales, alumbre intravaginal, piramidón en igual forma, óvulos de cebo, etc.

\section{Antecedentes}

Para este efecto se agruparon en 4 grandes grupos, así: Quirúrgicos, Obstétricos, Ginecológicos y Médicos. Los hallazgos se enumeran a continuación:

Quirúrgicos :

$$
\begin{aligned}
& \text { Nefrectomía } \\
& \text { Apendicectomía } \\
& \text { Colecistectomía } \\
& \text { Neovejiga }
\end{aligned}
$$

\section{Obstétricos :}

Cesáreas ( $\left.\begin{array}{llll}\text { Ee } & 1 & \text { a } & 6\end{array}\right)$
Eclampsia
RH negativo
Mola Hidatiforme

Ginecológicos :

Plastia vaginal

Peritonitis post-aborto

\author{
Ooforectomía \\ Embarazo extrauterino \\ Anexitis residual \\ Utero Bicorne \\ Ligamentopexia \\ Fistulorrafia \\ Miomectomía \\ Polipectomía \\ Condilomatosis \\ Carcinoma de seno \\ Bartolinitis
}

Médicos:

Psicosis maníaco-depresiva Asma

\section{Reacciones inmediatas}

Consideramos como tales aquellas manifestaciones relatadas por la paciente en el momento mismo de la inserción del dispositivo. El $90 \%$ de las pacientes no presentaron ninguna reacción considerándola como "fácil, indolora". El resto de las pacientes un $10 \%$ presentó manifestaciones de dolor o lipotimia más o menos severa que cedió a los analgésicos; no fué necesario retirarlo en ningún caso. 


\section{Reacciones Posteriores}

Consideradas como aquellas manifestaciones que presentan las pacientes en los días posteriores a la aplicación del dispositivo y antes del primer control, que se efectúa al mes. En su orden de incidencia son:

Metrorragias, más o menos severas en el $70 \%$ de las pacientes; no requirieron tratamiento y cedieron en los primeros 30 días.

Dolor, más o menos intenso comparable a un cólico menstrual y cedió a la ingestión de droga, tipo $\mathrm{Fe}$ nil-Butazona; se encontró en un 10\% de las pacientes. El síntoma flujo se presentó en un $60 \%$ de las pacientes, pero dada la alta incidencia en el mismo ingreso de las pacientes de este síntoma, no lo consideramos de valor, simplemente en controles posteriores a estas pacientes se les practicó un frotis vaginal y su tratamiento adecuado.

\section{Complicaciones}

Las complicaciones que se encontraron en la revisión de las 1.000 historias clínicas fueron en su orden de incidencia las siguientes:

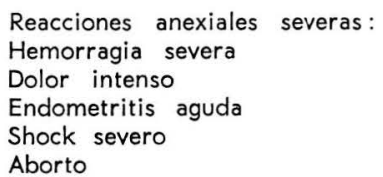

$\begin{array}{lc}7 & \text { casos } \\ 4 & \text { "' } \\ 1 & \prime \\ 1 & \prime \\ 1 & \prime \\ 1 & \prime \prime\end{array}$

Esta última complicación (Historia № 1828) se debió a engaño de la paciente en cuanto a la fecha de su última menstruación; esta paciente fué descubierta por el marido, que sabía de su estado de embarazo, muy reciente para ser detectado al examen, y su deseo de abortar, valiéndose de la inserción del IUD.

\section{Controles}

Revisando los controles a que estas 1.000 pacientes habían concurrido (hasta Dicbre. 31/66) se encontró que 991 pacientes, o sea el 99.1 habían asistido por lo menos a un control, lo cual puede ser considerado como un índice de la motivación que creemos se da a las pacientes en el Centro. Estos controles incluyen fecha de la última menstruación, estado general de la paciente y condiciones en que se encuentra el dispositivo o el método escogido.

\section{Expulsiones}

Cincuenta y un pacientes expulsaron el dispositivo dentro de los primeros cuatro meses de estudio (Agosto-Dicbre.), la mayor incidencia se notó al finalizar el tercer mes; corresponde al $5.1 \%$, fué reinsertado en la totalidad de las pacientes y fué reexpulsado en 10 pacientes, 1.5\%; como se puede ver el porcentaje de expulsión luego de la segunda inserción es menor, pudiéndose pensar en un defecto de técnica en la colocación inicial; también se observó que a mayor paridad hay menor expulsión del IUD. Es de anotar que la expulsión por tres veces consecutivas es indicación absoluta de cambio de método.

\section{Retiros}

Hasta la fecha de corte había sido necesario retirar el dispositivo en seis casos por los siguientes motivos:

Deseo de otro hijo:

Inseguridad:

Hemorragia irreversible:

Voluntario:

Dolor intenso:

$\begin{array}{ll}1 & \text { caso } \\ 1 & \text { "' } \\ 2 & \text { "' } \\ 1 & \text { "' }\end{array}$

\section{Embarazos}

En total se presentaron 5 embarazos comprobados, hasta Dicbre. 31, 
o sea, en pacientes que completaban cuatro meses de uso; esto reduce un tanto la evaluación correcta de la eficacia del IUD, que debe ser hecha por lo menos con 100 mujeres $y$ 1.200 ciclos de observación continua; tan solo se trata de dar un informe preliminar sobre estas pacientes. El porcentaje de fracaso pues, fué de $0.3 \%$.

NOTA: La observación de estos 5 casos reportó lo siguiente: 2 pacientes siguen en control prenatal; 2 pacientes se provocaron aborto, confesaron y volvieron al Centro para continuar usando el método y 1 paciente no volvió a control.

\section{Citología Vaginal}

Se incluye este dato al final del análisis, ya que los datos iniciales no pudieron ser obtenidos en vista de que aún no se practicaba Citología de rutina en todas las usuarias, tan solo en el mes de Enero de 1967 se inició el programa de Diagnóstico Precoz del Cáncer en una forma regular; hoy podemos asegurar que el $88.5 \%$ de estas pacientes están controladas en dicho programa y es seguro que el resto de las usuarias estarán cubiertas al finalizar este año. 